\title{
A second-order conditional moment closure model for the simulation of a lifted turbulent flame
}

\author{
C. B. Devaud*
}

(Received 8 August 2003, revised 28 January 2004)

\begin{abstract}
This paper presents the results of a second-order closure Conditional Moment Closure model applied to a lifted turbulent $\mathrm{H}_{2}$-air flame. The objective is to find out how a second-order closure will modify the lift-off height compared to first-order results. A 10-step chemical mechanism is used to predict rates of reaction in hydrogen-air mixtures but the second-order correction terms are calculated using one-step chemistry. First a progress variable for this reduced mechanism is defined and an additional equation for its conditional variance is derived. The level of the conditional fluctuation is found to be around $30 \%$ of its maximum value for stoichiometric mixtures in the
\end{abstract}

*School of Aerospace, Mechanical and Mechatronic Engineering, University of Sydney, Australia. Currently: Department of Mechanical Engineering, University of Waterloo, N2L 3G1, CANADA mailto: cdevaud@mecheng1. uwaterloo.ca

See http://anziamj.austms.org.au/V45/CTAC2003/Deva for this article, (C) Austral. Mathematical Soc. 2004. Published June 10, amended June 11, 2004. ISSN 1446-8735 
flame stabilisation area. The second-order closure has a distinct effect on the flame position determined in the simulations.

\section{Contents}

1 Introduction

C420

2 Governing equations

C421

3 Numerical Method

$\mathrm{C} 427$

4 Results

C429

5 Conclusion

C433

References

C433

\section{Introduction}

The crucial issue in turbulent combustion modelling remains the closure of the mean chemical source term in the averaged species transport equations. A detailed review of the existing strategies for modelling chemistry-turbulence interactions is beyond the scope of this paper. Instead, this study is focused on a particular method for turbulent non-premixed flames. Conditional Moment Closure (CMC) has been independently developed by Klimenko [8] and Bilger [1]. The idea is to solve transport equations for reactive species mass fractions which are conditionally averaged on a conserved scalar. The main advantage is that detailed chemistry can be accounted for at low computational cost.

The first-order CMC model assumes that the conditional fluctuations are 
negligible and that the conditional chemical source term is only a function of the first-order moments, that is, the conditional species concentrations and temperature. This closure assumption has been successfully applied in several cases of turbulent non-premixed combustion, but may be inaccurate for flame stabilisation problems. One possible improvement is to close the conditional chemical source term using a second-order approximation [9].

The present study is based on previous calculations using a first-order closure for a lifted turbulent flame [3]. Here, the flame base is detached from the fuel duct, and combustion takes place further downstream. The lift-off height is defined as the distance between the burner exit plane and the flame base. Figure 1 shows the experimental configuration. The lift-off height was reasonably well predicted compared with the experimental value [3], but the magnitude and the effect of the conditional fluctuations had not been determined. It is now proposed to implement a second-order closure method for the same flame and study the impact on the flame position.

\section{Governing equations}

The instantaneous mass fraction of species is decomposed as:

$$
Y_{i}=Q_{i}+Y_{i}^{\prime \prime} ; \quad Q_{i} \equiv\left\langle Y_{i} \mid \eta\right\rangle .
$$

In the present derivation, the conditioning variable is the mixture fraction $Z$, bounded between 0 (pure air) and 1 (pure fuel). $\langle. \mid \eta\rangle$ denotes ensemble averaging subject to the condition that $Z=\eta$. $Y_{i}^{\prime \prime}$ is the fluctuation with respect to the conditional mean, $Q_{i}$. The resulting CMC equation for the flame configuration shown in Figure 1, assuming steady-state and axi-symmetric 


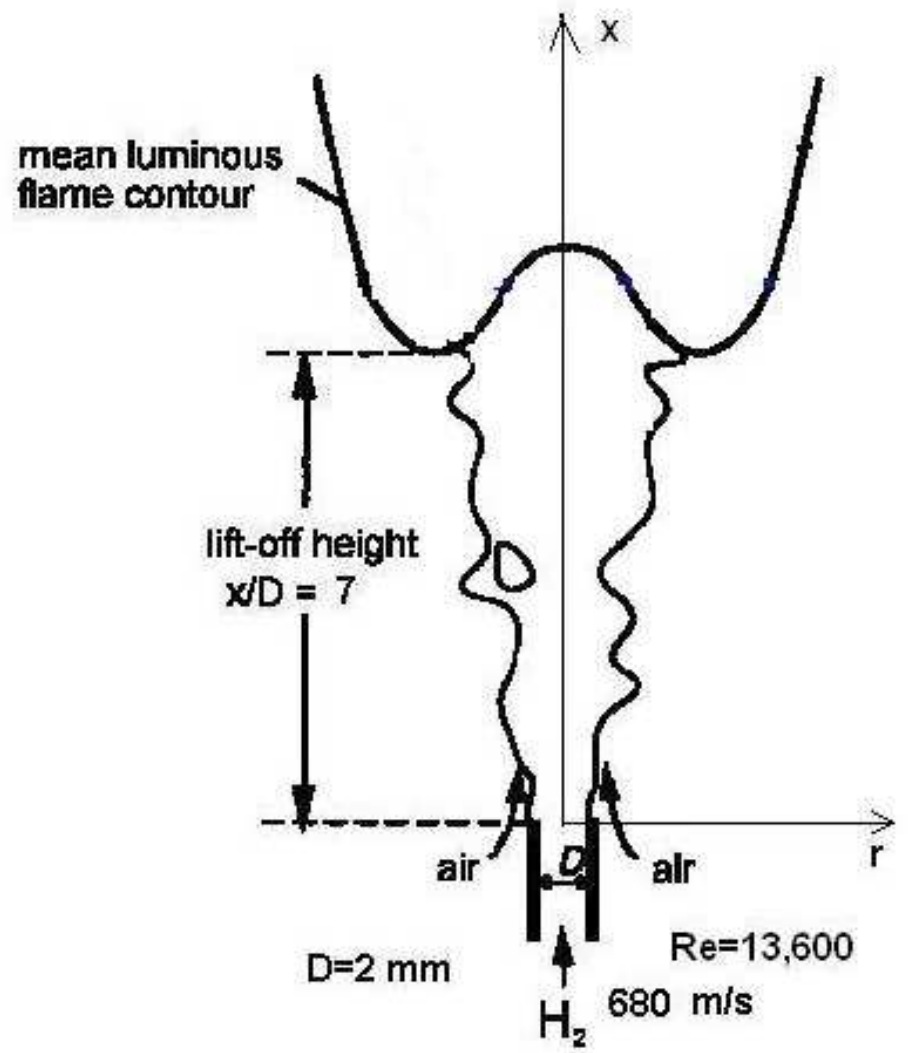

FiguRE 1: Experimental configuration 
flow, is [3]:

$$
\begin{aligned}
& \left(\left\{\left\langle V_{x} \mid \eta\right\rangle\right\}_{R+}-D_{t} \frac{1}{\tilde{P}(\eta)} \frac{\partial \tilde{P}(\eta)}{\partial x}\right) \frac{\partial Q_{i}}{\partial x}-\frac{\partial}{\partial x}\left(\left\{D_{t} \mid \eta\right\}_{R+} \frac{\partial Q_{i}}{\partial x}\right) \\
= & \left\langle\dot{\omega}_{i} \mid \eta\right\rangle \frac{W_{i}}{\langle\rho \mid \eta\rangle}+\frac{1}{2}\{\langle\chi \mid \eta\rangle\}_{R+} \frac{\partial^{2} Q_{i}}{\partial \eta^{2}},
\end{aligned}
$$

with $V_{x}$ being the axial velocity, $D_{t}$ the turbulent diffusivity, $\tilde{P}(\eta)$ the Favre averaged probability density function (PDF) for $\eta, \dot{\omega}_{i}$ the chemical production of species $i, \chi(\chi \equiv 2 D \nabla Z \cdot \nabla Z)$ the scalar dissipation rate, with $D$ being the molecular diffusivity of $Z, W_{i}$ the molecular weight of species $i$ and $\rho$ the density. Equation (2) is solved in the axial direction $x$ and in mixture fraction space, $\eta$, but is cross-stream averaged in the radial direction $r$ following:

$$
\{M\}_{R} \equiv \frac{2}{R^{2}} \int_{0}^{R} M(r) r d r ; \quad\{M\}_{R+}=\frac{\{M(r) \tilde{P}(\eta)\}_{R}}{\{\tilde{P}(\eta)\}_{R}} .
$$

$R$ is a bounding radius chosen to be as large as possible.

The closure of $\left\langle V_{x} \mid \eta\right\rangle, D_{t}$ and $\langle\chi \mid \eta\rangle$ was previously shown [3] and is not repeated here. The focus of this paper is on the closure of the chemical source term, $\left\langle\dot{\omega}_{i} \mid \eta\right\rangle$. In order to include the second moments in the calculation of the chemical source term, the variances and covariances of the conditional moments must be determined.

Solving the conditional variances for a realistic chemical mechanism can result in a significant increase in the number of equations, that is, $(n+1)(n+$ 2)/2 additional equations would need to be solved for a system of $n$ species. For a hydrogen-air system, the leading order of the reaction rates may be computed using a full mechanism whilst the second-order corrections to these rates may be related to one progress variable, $\Gamma_{t}$, characterising the chemical system:

$$
\Gamma_{t}=\sum_{i=1}^{n} \frac{Y_{i}}{W_{i}} ; \quad\left\langle\Gamma_{t} \mid \eta\right\rangle=\sum_{i=1}^{n} \frac{Q_{i}}{W_{i}} .
$$


It is here assumed that the chemistry is simplified to a one-step reaction based on partial-equilibrium for the fast-two-body-reactions [2], that is, the forward rate and the backward rate for a reaction $k$ are equal. In Table 1 the index $f$ stands for forward and $b$ for backward. In the rest of the paper, the superscript (pe) will mean that partial equilibrium assumptions are used. Differential diffusion is neglected and no radiation is considered. The allowable maxima $\left(\Gamma_{\max }(\eta)\right)$ occur when fuel and air are mixed without reaction and the allowable minima occur $\left(\Gamma_{\min }(\eta)\right)$ when combustion is completed and reaches the equilibrium state. Figure 2 shows these two bounds in $\eta$ space. The conditional expectations of the second moments of $\Gamma_{t}$ are defined as:

$$
K \equiv\left(\Gamma_{t}^{\prime \prime}\right)^{2} ; \quad G \equiv\langle K \mid \eta\rangle
$$

The derivation of the transport equation for $G$ is not reproduced here but can be found in [6]. The governing equation for $G$ assuming a high Reynolds number, steady-state conditions and using cross-stream averages is

$$
\begin{gathered}
\left\{\left\langle V_{x} \mid \eta\right\rangle\right\}_{R+} \frac{\partial G}{\partial x}-\frac{1}{2}\{\langle\chi \mid \eta\rangle\}_{R+} \frac{\partial^{2} G}{\partial \eta^{2}}+\left\{\frac{\operatorname{div}\left(\left\langle\mathbf{v}^{\prime \prime} K^{\prime \prime} \mid \eta\right\rangle P(\eta)\langle\rho \mid \eta\rangle\right)}{P(\eta)\langle\rho \mid \eta\rangle}\right\}_{R+} \\
=2\left\langle\dot{\omega}_{\Gamma_{t}}^{\prime \prime} \Gamma_{t}^{\prime \prime} \mid \eta\right\rangle-2\left\{\left\langle D\left(\nabla \Gamma_{t}^{\prime \prime}\right)^{2} \mid \eta\right\rangle\right\}_{R+}+\frac{\partial^{2}\left\langle\Gamma_{t} \mid \eta\right\rangle}{\partial \eta^{2}}\left\{\left\langle\Gamma_{t}^{\prime \prime} \chi^{\prime \prime} \mid \eta\right\rangle\right\}_{R+}+\left\{F_{G}\right\}_{R+} \cdot
\end{gathered}
$$

On the left-hand-side (LHS) the first term represents axial advection of $G$ at constant $\eta$, the second term is a diffusive transport of $G$ and the third term is the conditional turbulent flux generated by the fluctuations of the velocity and of the reactive scalars. On the right-hand-side (RHS), the first term is the chemical contribution, which can be positive or negative, the second term is a sink due to the dissipation of the conditional correlation, the third is a production term due to scalar dissipation-scalar fluctuations and $F_{G}$ is a turbulent transport term. In Equation (6) the PDF $P$ is not Favre averaged and the relationship $\langle\rho \mid \eta\rangle P(\eta)=\langle\rho\rangle \tilde{P}(\eta)$ is then used in Equation (11). The 


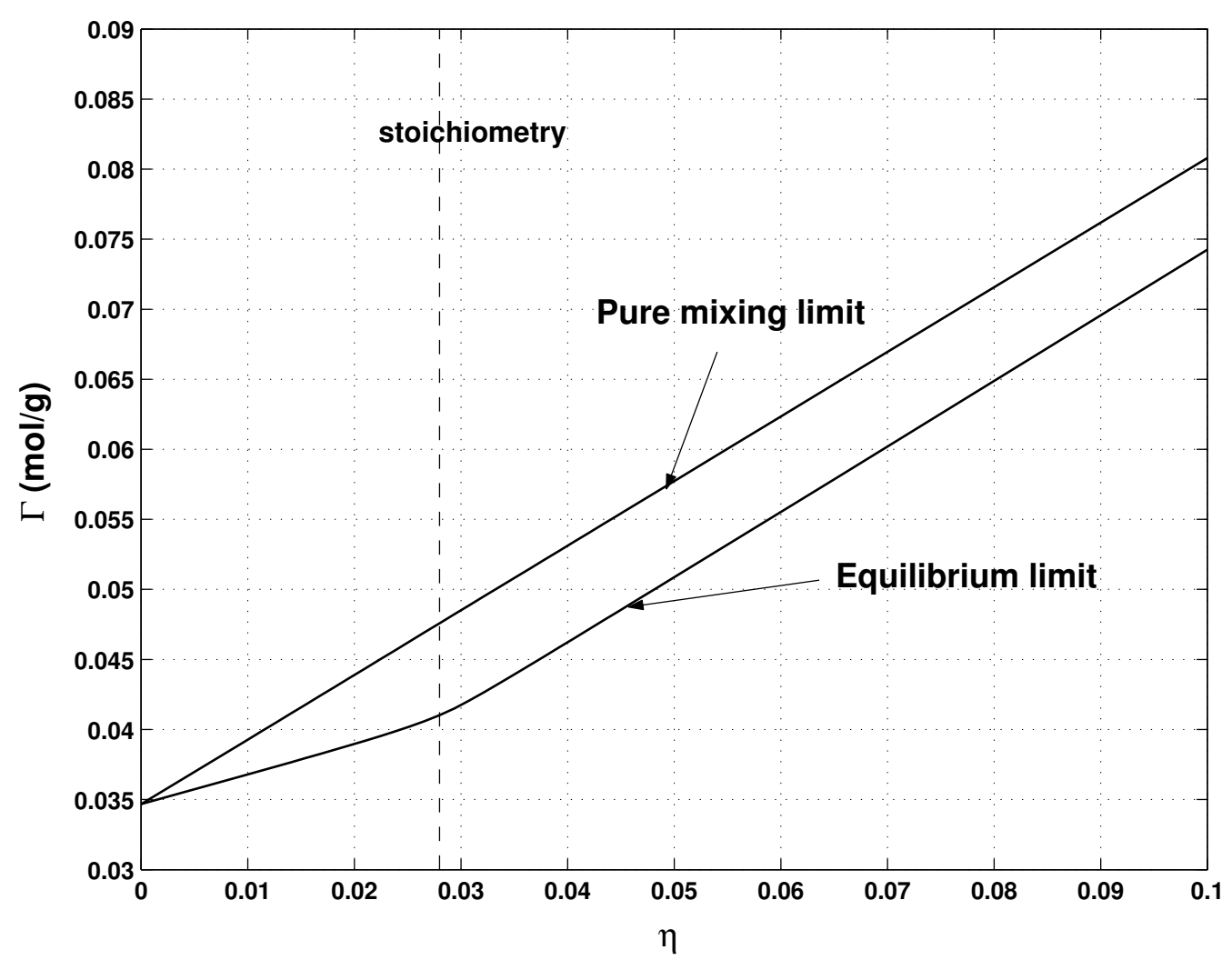

Figure 2: Integration domain of $\Gamma$ in $\eta$ space $(0 \leq \eta \leq 1)$. For clarity, only values between $0 \leq \eta \leq 0.1$ are shown. 
chemical fluctuation term is modelled [9]:

$$
\begin{aligned}
\left\langle\dot{\omega}_{\Gamma_{t}}^{\prime \prime} \Gamma_{t}^{\prime \prime} \mid \eta\right\rangle= & \left\langle\dot{\omega}_{\Gamma_{t}} \Gamma_{t} \mid \eta\right\rangle-\left\langle\dot{\omega}_{\Gamma_{t}} \mid \eta\right\rangle\left\langle\Gamma_{t} \mid \eta\right\rangle \\
\approx & \int_{\Gamma_{t}} \dot{\omega}_{\Gamma_{t}}^{p e}\left(\Gamma_{t}, \eta\right) \Gamma_{t} P\left(\Gamma_{t}\right) d \Gamma_{t} \\
& -\left\langle\Gamma_{t} \mid \eta\right\rangle \int_{\Gamma_{t}} \dot{\omega}_{\Gamma_{t}}^{p e}\left(\Gamma_{t}, \eta\right) P\left(\Gamma_{t}\right) d \Gamma_{t} .
\end{aligned}
$$

Here $\dot{\omega}_{\Gamma_{t}}$ is the formation/destruction rate of $\Gamma_{t}$. The PDF selected is a $\beta$ PDF based on $\left\langle\Gamma_{t} \mid \eta\right\rangle$ and $\left\langle\Gamma_{t}^{\prime \prime 2} \mid \eta\right\rangle$. Similarly, a presumed $\beta$ form for the PDF of $\eta$ based on the mean, $\tilde{Z}$, and the variance, $\widetilde{Z^{\prime \prime 2}}$ is also used. A correction term is calculated for each reaction step $k$ using

$$
\dot{\omega}_{k, \mathrm{corr}}^{p e}=\left\langle\omega^{p e}{ }_{k} \mid \eta\right\rangle-\dot{\omega}_{k}^{p e}\left(\left\langle\Gamma_{t} \mid \eta\right\rangle, \eta\right) .
$$

The chemical source term in Equation (2) is calculated by adding a secondorder correction to the first-order closure.

$$
\left\langle\dot{\omega}_{k} \mid \eta\right\rangle=\underbrace{\dot{\omega}_{k}\left(Q_{i}, Q_{T}\right)}_{\text {1st-order }}+\underbrace{\dot{\omega}_{k, \text { corr }}^{p e}}_{\text {2nd-order }} .
$$

with $Q_{T}$ being the conditional temperature. The closure of the remaining terms is achieved following $[3,11,7,9]$. This yields:

$$
\begin{aligned}
& \left(\left\{V_{x} \mid \eta\right\}_{R+}-D_{t} \frac{1}{\tilde{P}(\eta)} \frac{\partial \tilde{P}(\eta)}{\partial x}\right) \frac{\partial G}{\partial x}-\frac{\partial}{\partial x}\left(\left\{D_{t} \mid \eta\right\}_{R+} \frac{\partial G}{\partial x}\right) \\
= & \frac{1}{2}\{\chi \mid \eta\}_{R+} \frac{\partial^{2} G}{\partial \eta^{2}} \\
& -\left[\frac{\partial \log c^{S}}{\partial \eta} \frac{\partial \log c^{S}}{\partial \eta}\right]\{\langle\chi \mid \eta\rangle\}_{R+}\{G\}_{R+} \\
& +R_{T}\{\chi \mid \eta\}_{R+} G^{1 / 2} \frac{\partial^{2}\left\langle\Gamma_{t} \mid \eta\right\rangle}{\partial \eta^{2}}+2\left\langle\dot{\omega}_{\Gamma_{t}}^{\prime \prime} \Gamma_{t}^{\prime \prime} \mid \eta\right\rangle \\
& +\frac{1}{\{P(\eta)\}_{R}} \frac{\partial}{\partial \eta}\left(\frac{1}{2}\{\langle\chi \mid \eta\rangle\}_{R+}\{P(\eta)\}_{R+} \frac{\partial G}{\partial \eta}\right)
\end{aligned}
$$


TABLE 1: 10-step reaction mechanism — Units in Moles, Kelvin, Seconds, Cubic Centimetres and Calories per Mole-Third body coefficients: $\mathrm{H}_{2} \mathrm{O}$ 6.5, $\mathrm{H}_{2}$ 1.0, $\mathrm{O}_{2}$ 0.4, $\mathrm{N}_{2}$ 0.4, all other species 1.0 [10], A, b and $\mathrm{E}$ are the usual reaction rate parameters

\begin{tabular}{|c|c|c|c|c|}
\hline & Reaction & $\mathrm{A}$ & $\mathrm{b}$ & $\mathrm{E}$ \\
\hline $1 \mathrm{f}$ & $\mathrm{H}+\mathrm{O}_{2} \rightarrow \mathrm{OH}+\mathrm{O}$ & $2.0 \times 10^{14}$ & 0.0 & 16802.1 \\
\hline $1 b$ & $\mathrm{O}+\mathrm{OH} \rightarrow \mathrm{H}+\mathrm{O}_{2}$ & $1.57 \times 10^{13}$ & 0.0 & 841.3 \\
\hline $2 \mathrm{f}$ & $\mathrm{H}_{2}+\mathrm{O} \rightarrow \mathrm{OH}+\mathrm{H}$ & $5.06 \times 10^{4}$ & 2.67 & 6285.8 \\
\hline $2 b$ & $\mathrm{OH}+\mathrm{H} \rightarrow \mathrm{H}_{2}+\mathrm{O}$ & $2.22 \times 10^{4}$ & 2.67 & 4371.4 \\
\hline $3 f$ & $\mathrm{H}_{2}+\mathrm{OH} \rightarrow \mathrm{H}_{2} \mathrm{O}+\mathrm{H}$ & $1.00 \times 10^{8}$ & 1.60 & 3298.3 \\
\hline $3 \mathrm{~b}$ & $\mathrm{H}+\mathrm{H}_{2} \mathrm{O} \rightarrow \mathrm{H}_{2}+\mathrm{OH}$ & $4.31 \times 10^{8}$ & 1.60 & 18274.4 \\
\hline $4 \mathrm{f}$ & $\mathrm{OH}+\mathrm{OH} \rightarrow \mathrm{H}_{2} \mathrm{O}+\mathrm{O}$ & $1.50 \times 10^{9}$ & 1.14 & 100.4 \\
\hline $4 \mathrm{~b}$ & $\mathrm{H}_{2} \mathrm{O}+\mathrm{O} \rightarrow \mathrm{OH}+\mathrm{OH}$ & $1.473 \times 10^{10}$ & 1.14 & 16990.9 \\
\hline 5 & $\mathrm{H}+\mathrm{HO}_{2} \rightarrow \mathrm{OH}+\mathrm{OH}$ & $1.50 \times 10^{14}$ & 0.0 & 1004 \\
\hline 6 & $\mathrm{H}+\mathrm{HO}_{2} \rightarrow \mathrm{H}_{2}+\mathrm{O}_{2}$ & $2.50 \times 10^{13}$ & 0.0 & 700 \\
\hline 7 & $\mathrm{OH}+\mathrm{HO}_{2} \rightarrow \mathrm{H}_{2} \mathrm{O}+\mathrm{O}_{2}$ & $2.00 \times 10^{13}$ & 0.0 & 1000.0 \\
\hline 8 & $\mathrm{H}+\mathrm{O}_{2}+\mathrm{M} \rightarrow \mathrm{HO}_{2}+\mathrm{M}$ & $2.30 \times 10^{18}$ & -0.8 & 0.0 \\
\hline 9 & $\mathrm{OH}+\mathrm{H}+\mathrm{M} \rightarrow \mathrm{HO}_{2}+\mathrm{M}$ & $2.20 \times 10^{22}$ & -2.0 & 0.0 \\
\hline 10 & $H+H+M \rightarrow H_{2}+M$ & $1.80 \times 10^{18}$ & -1.0 & 0.0 \\
\hline
\end{tabular}

with $c^{S}$ is the conditional fluctuation approximated by the steady laminar flamelet model [11] and $R_{T}$ is taken as a constant equal to 1.1 [9].

\section{Numerical Method}

Applying partial equilibrium for reactions 1-4 (see Table 1), steady-state for $\mathrm{HO}_{2}$ and considering the conservation of enthalpy and atoms results in solving a system of 9 nonlinear equations for a given value of $\eta$ and of $\Gamma_{t}$. 
This system of stiff equations is solved by a Newton-Raphson method. The chemistry is then tabulated, that is, $\dot{\omega}_{\Gamma_{t}}^{p e}\left(\eta, \Gamma_{t}\right)$ and $\dot{\omega}_{k}^{p e}\left(\eta, \Gamma_{t}\right)$ as functions of $\eta$ and $\Gamma_{t}$ for the integrations in Equation (8) and Equation (9). Note that the partial equilibrium assumptions are only valid for temperatures higher than $1200 \mathrm{~K}$. It was therefore necessary to impose a profile between the value of $\Gamma_{t}$ corresponding to $1200 \mathrm{~K}$ and its maximum value $\Gamma_{\max }$ (non-burning conditions). At the two limits, in $\Gamma_{t}$ space, $\dot{\omega}^{p e} \rightarrow 0$. In between these two bounds, the magnitude of the reaction rates are expected to increase, to reach a peak and then to decrease to 0 when $\Gamma_{t}$ is equal to $\Gamma_{\max }[2]$. A scaled symmetry is applied; hence the added values in the second part of the tabulations exhibit a similar profile to those obtained by Newton-Raphson. Figure 3 shows the tabulated values used for $\dot{\omega}_{\Gamma_{t}}^{p e}$ at $\eta=0.028$.

Equation (2) and Equation (11) are coupled and solved sequentially: Equation (2) is first solved for 7 reactive species, in the axial direction (x) and in mixture fraction space $\eta$. Then, $G$ is determined in the computational domain using Equation (11). The chemical source term is updated in Equation (2), which is solved again and so forth. Equation (2) and Equation (11) are solved using a time splitting method in which axial transport is calculated first, then transport in $\eta$ space and followed by the chemical source term. Information from the velocity and scalar field is needed in Equation (2) and Equation (11). In this study, the turbulent flowfield and the combustion modelling are computed separately [3]. Steady-state solutions are here of interest. Convection is approximated by a first-order upwind scheme, diffusion by a second-order central differencing scheme and the resulting systems of equations is solved using either by LU decomposition method for the first step, or by LSODE [5] in non-stiff/stiff conditions, respectively, for the last two steps. The first two steps are fully implicit in time. The computational domain includes 54 nodes in mixture fraction space, unevenly distributed. In the axial direction, the domain extends from the nozzle exit $\left(x_{0}\right)$ to $0.03 \mathrm{~m}$ $\left(x_{\max }\right)$. Successive refinements were performed in order to capture the high spatial gradients. The final mesh comprises 140 nodes with a grid spacing of $0.04 \mathrm{~mm}$ at its smallest value. At $\eta=1$ only pure fuel is present and 
at $\eta=0$ only air is found. At the nozzle exit, non-reacting profiles are set for $Q_{i}$. At the end of the physical domain, the mixture is set to be fully burning. For the conditional variance, $G(x, \eta=1)=0, G(x, \eta=0)=0$, $G\left(x_{0}, \eta\right)=10^{-8} \mathrm{~mol}^{2} \mathrm{~kg}^{-2}$ and $G\left(x_{\max }, \eta\right)=10^{-8} \mathrm{~mol}^{2} \mathrm{~kg}^{-2}$. The results presented in the next section are shown to be grid-independent.

\section{Results}

Figure 4 presents the axial profile of the conditional temperature at stoichiometry, compared to the first-order results. The flame position is determined by using the $1000 \mathrm{~K}$-value, which is close to the autoignition temperature for $\mathrm{H}_{2}$ /air mixtures. The stoichiometric mixture is selected because it corresponds to the most chemically reactive conditions. For clarity, the experimental value for the flame stabilisation position was $14 \mathrm{~mm}$ [3]. As can be seen, the flame moves further downstream when the conditional fluctuations are included in the CMC model. This is very encouraging compared to the most recent fully-coupled CMC calculations of lifted turbulent flames where the flame position was shown to be severely underpredicted [4]. However, the relative change between the first and second-order results remains small, in the order of $10 \%$.

Figure 5 shows the axial profile of the normalised conditional variance at the stoichiometric mixture fraction. $G$ is normalised by its maximum attainable value $G_{\max }$, defined as $\left(\left\langle\Gamma_{t} \mid \eta\right\rangle-\Gamma_{\min }(\eta)\right)\left(\Gamma_{\max }(\eta)-\left\langle\Gamma_{t} \mid \eta\right\rangle\right)$. The normalised fluctuations gradually increase in the flame stabilisation area. The maximum of the normalised conditional variance occurs just upstream of the lift-off height, with a level of around $35 \%$. Then the profile declines as the flame is spreading downstream towards the chemical equilibrium state. High values of $G$ in the stabilising region of the flame characterise the intermittent state between burnt and unburnt mixtures and shows that local extinction and ignition take place. The high values of $G$ would be expected to produce 


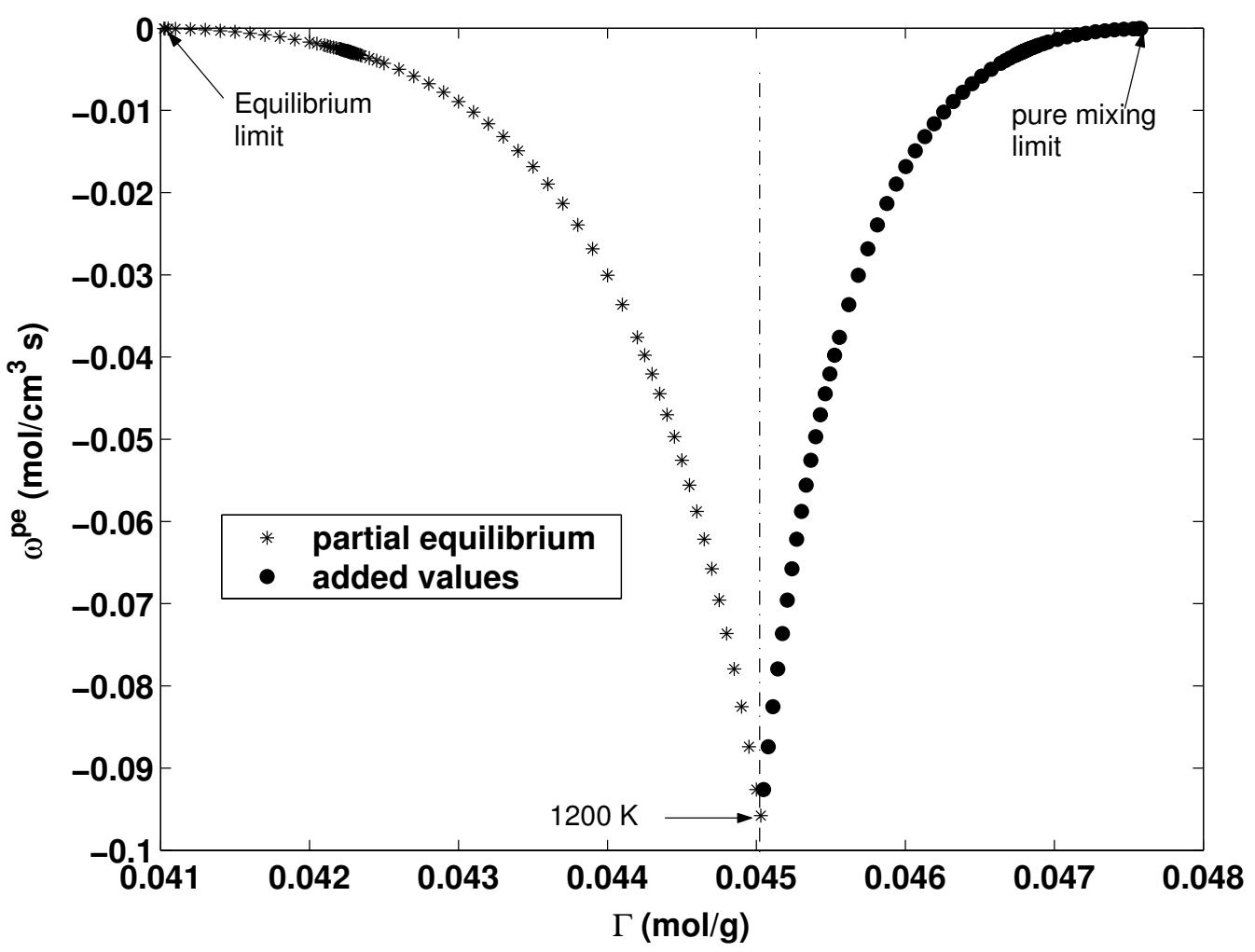

Figure 3: Tabulated values of $\omega_{\Gamma_{t}}^{p e}$ as a function of $\Gamma_{t}$ at $\eta=0.028$ (stoichiometric mixture). 


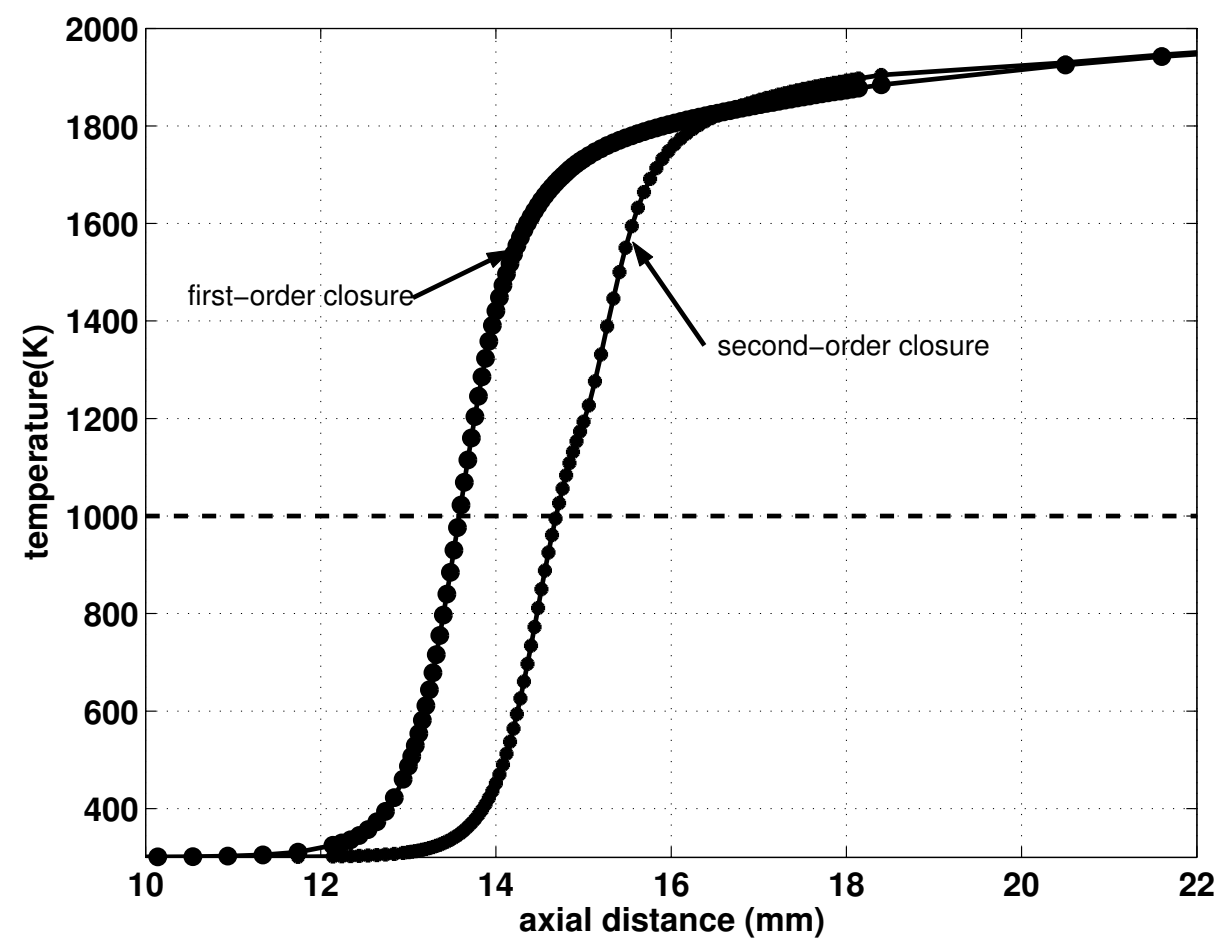

Figure 4: Axial profiles at stoichiometric mixtures: Temperature axial profile at $\eta=0.028$. 


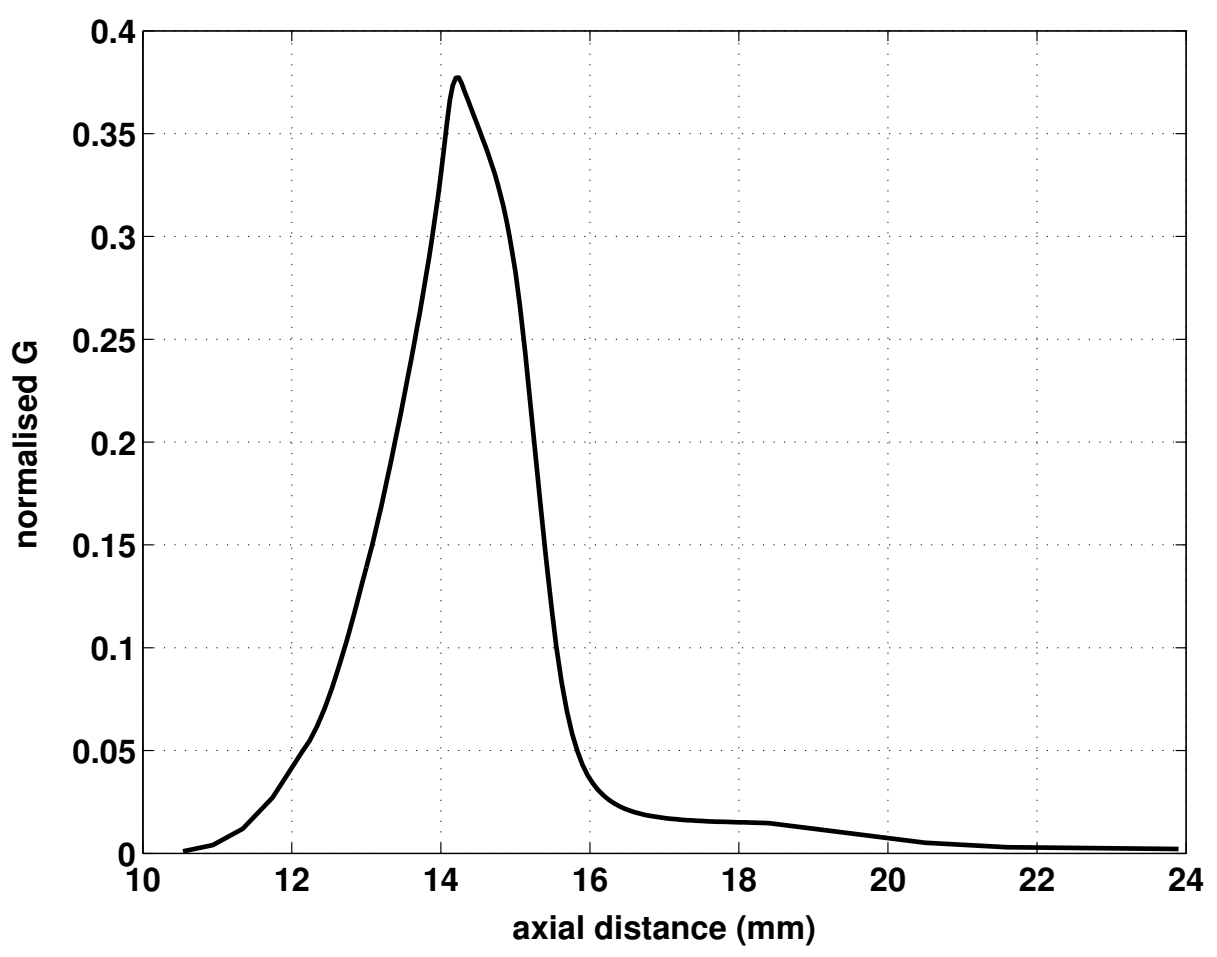

Figure 5: Axial profiles at stoichiometric mixtures: Normalised G axial profile at $\eta=0.028$. 
a large effect on the species production rates, much larger than $10 \%$. The most plausible explanation is that the the two-body-reactions are assumed to be in partial equilibrium and are therefore not corrected by the current second-order closure method. In particular, reaction $1 \mathrm{f}$ appears to be critical in the rate of formation/destruction of $H$ radicals.

\section{Conclusion}

A second-order conditional method has been successfully applied to a lifted turbulent flame. The results show that there is a significant level of conditional fluctuations around the flame base. In this case, a second-order closure is justified. The lift-off height increases when the second-order closure is used. This is a very positive result compared to previous first order calculations. At this stage the correction terms appear to be too small. The most probable reason is that the two-body-reactions are not corrected by the current method. A two-step-simplified-mechanism is being investigated where no partial equilibrium assumptions are used.

Acknowledgement: This work was supported by the Australian Research Council.

\section{References}

[1] R. W. Bilger. Conditional moment closure for turbulent reacting flow. Phys. Fluids A, 5(2):436-444, 1993. C420

[2] J-Y. Chen and W. Kollmann. Chemical Models for PDF Modeling of Hydrogen-Air Nonpremixed Turbulent Flames. Combust. Flame, 79:75-99, 1990. C424, C428 
[3] C. B. Devaud and K. N. C. Bray. Assessment of the Applicability of Conditional Moment Closure to a Lifted Turbulent Flame : First Order Model. Combust. Flame, 132:102-114, 2003. C421, C423, C426, C428, C429

[4] C. B. Devaud, J. H. Kent, and R. W. Bilger. Conditional Moment Closure applied to a lifted turbulent methane-air flame. Proc. Med. Combust. Symp., 3:972-981, 2003. C429

[5] A. C. Hindmarsh. ODEPACK, a systemized collection of ODE solvers. In R. S. Stepleman et al., editor, Scientific Computing, pages 55-64. North Holland, Amsterdam, 1983. C428

[6] S. H. Kim. On the Conditional Variance and Covariance Equations for second-Order Conditional Moment Closure. Phys. Fluids, 14(6):2011-2014, 2002. C424

[7] S. H. Kim, K. Y. Huh, and R. W. Bilger. Second-Order Conditional Moment Closure Modeling of Local Extinction and Reignition in Turbulent Nonpremixed Hydrocarbon Flames. Proc. Combust. Inst. (To appear), 29, 2002. C426

[8] A. Yu. Klimenko. Multicomponent diffusion of various admixtures in turbulent flow. Fluid Dyn., 25,37, 1990. C420

[9] A. Kronenburg, R. W. Bilger, and J. H. Kent. Second Order Conditional Moment Closure for Turbulent Jet Diffusion Flames. Proc. Combust. Inst., 27:1097-1104, 1998. C421, C426, C427

[10] C. J. Montgomery, G. Kosály, and J. J. Riley. Direct Numerical Solutions of Turbulent Nonpremixed Combustion with Multistep Hydrogen-Oxygen Kinetics. Combust. Flame, 109:113-144, 1997. C427

[11] N. Swaminathan and R. W. Bilger. Study of the conditional covariance and variance equations for second order conditional moment closure. Phys. Fluids, 11(9):2679-2695, 1999. C426, C427 